\title{
Cloud Computing: Technical, Non-Technical and Security Issues
}

\author{
Wada Abdullahi \\ Federal College of Education \\ (Technical) \\ Potiskum \\ Yobe State, Nigeria
}

\author{
Alhaji Idi Babate \\ Federal College of Education \\ (Technical) \\ Potiskum \\ Yobe State, Nigeria
}

\author{
Ali Garba Jakwa \\ Federal College of Education \\ (Technical) \\ Potiskum \\ Yobe State, Nigeria
}

\begin{abstract}
Cloud Computing has been growing over the last few years as a result of cheaper access to high speed Internet connection and many applications that comes with it. Its infrastructure allows it to provide services and applications from anywhere in the world. However, there are numerous technical, non-technical and security issues that come with cloud computing. As cloud computing becomes more adopted in the mainstream, these issues could increase and potentially hinder the growth of cloud computing. This paper investigates what cloud computing is, the technical issues associated with this new and evolving computing technology and also the practical applications of it. It also addresses and highlight the main security issues in cloud computing.
\end{abstract}

Keywords: computing; technical; security; cloud; issues

\section{INTRODUCTION}

Cloud Computing is a broad term used to describe the provision and delivery of services that are hosted over the internet [24]. The "cloud" in cloud computing comes the diagram representation or symbol that is usually used to depict the internet in network diagrams or flowcharts [24]; [19].

In this type of computing, servers on the internet store information permanently and temporarily cached on the client-side devices - including laptops, desktops, hand-held devices, monitors, sensors, tablet computers etc [19]. The infrastructure allows services to be provided and accessed from anywhere in the world as services are offered through data centres - virtually [19]. Here, the cloud becomes the single access point for customers/users to access services. This means that at any given time, a user has as little or as much of a service provided by the particular service provider. However, the user only needs a computer or appropriate device and Internet connection to gain access to the service(s).

In the cloud computing model, there are three main entities as illustrated in Figure 1 - End Users, Application Providers and Cloud Providers. According to [9], the assemble and organisation of users and computing resources "provides significant benefits to all three entities because of the increased system efficiency and availability."

There are numerous reason why cloud computing has gained interest over the last few years. One reason has been the significant and improved innovations in distributed and virtualisation computing. Additionally, the cost-benefits and access to high-speed internet have also contributed to this accelerated interest.

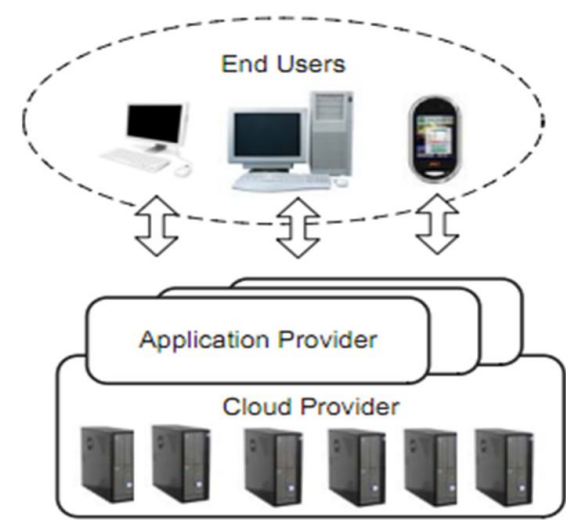

Figure 1: User of a cloud-based application system.

Cloud Providers provide the necessary cloud infrastructure which includes network facilities, replications sites and data centres. Applications services used on the cloud infrastructure are provided by Application Providers. These application services are then used by the "End Users".

\section{CLOUD COMPUTING MODELS}

The model of cloud computing can be divided into private or public. In public cloud, the providers of the cloud sell services to anyone, whereas in private cloud data centres or cloud providers they hosted their services only to a limited or small number of subscribers or buyers. However, there are situations where a service provider uses the resources of a public cloud to provide or create a private cloud - this is what is known as virtual private cloud [24]. In the service provision part of cloud computing, there are three main categories of service provision namely Software-as-a-Service (SaaS), Platform-as-a-Service (PaaS) and Infrastructure-as-aService (IaaS). 
In SaaS cloud model, the hardware infrastructure and the provision of the software product is supplied by the service provider supplies. Additionally, the user interacts with the service provider through a front-end portal [24]. In other words, SaaS can be said to be software that is "owned, delivered, and managed remotely by one or more providers." [8]. There are many companies that use business applications that are provided remotely [3]. According to Biddick, companies benefit from using SaaS as that development and maintenance of software applications becomes the burden of the provider.

In cloud computing, PaaS is described as "a set of software and product development tools hosted on the provider's infrastructure." This allows developers to develop and create software tools over the internet and also on the provider's platform. The use of APIs (Application Programming Interfaces), a gateway software and website portal on the end user's computer is commonly used by PaaS providers - this allows end users to develop software applications. However, data portability and standards for interoperability are not currently being used in cloud computing [24]. This makes it difficult to systems to work together or easy exchange of information.

In IaaS, providers "provides virtual server instance API to start, stop, access and configure their virtual servers and storage" [24]. End users can pay for the only capacity they require - making it possible to keep cost to a minimum for new start-up businesses. An example of an IaaS is Amazon Web Services - currently the largest provider [15].

\section{SOFTWARE EVOLUTION OF CLOUD COMPUTING}

The process of software evolution can be seen as a neverending process. Once software is developed, it is maintained, and then repeatedly updated with respect to changes in requirements, processes and methodologies. It is known that $90 \%$ of companies' software budget is spent on maintenance and adapting existing software tools than developing new ones from scratch [5].

Cloud computing is a specialised distributed computing on a large-scale [7]. However, there are differences from the traditional distrusted systems in many ways;

$\checkmark \quad$ Scalability is massive on cloud computing.

$\checkmark$ Different types or levels of services can be provided to clients outside the cloud with a greater degree of encapsulation.

$\checkmark$ Economies of scale is one of the main drivers of cloud computing.

$\checkmark$ Configuration of services is dynamic and delivery of services can be on demand.

The idea that evolution is driven by change can be observed in cloud computing. There is a growing demand for computing and storage problems in the so called "Internet Age". As a result many companies and individuals are looking to cloud computing to provide the answer [7].

The evolution of cloud computing could be traced back to the 1990s when Grid Computing was used to describe the collection of technologies that enabled users to obtained computing power when required. This lead to the standardisation of protocols to be allows for data exchange over the grid. However, according to the commercial utility of grid computing was very limited until about 2007/8 [7]. The vision of both cloud and grid computing technologies remains the same i.e. reduce computing cost with increased reliability, and transform the old style of standalone software computing to one where services can be obtained from third parties via the Internet. The underlying technologies and procedures of cloud and grid computing are somehow different.

Utility computing is a model based on the concept of demand and outsourcing availability [2]. In this type of model, resources and services are provided to the end user and charged based on usage.

The increasing demand for computing comes from our need to analyse large collection of data - data that was not present as of ten years ago. Additionally, there has been the realisation that operating mainframe computers are very expensive compared to commodity clusters. This has lead to a reduced cost of virtualisation. Over the last ten years, companies like Google, Microsoft and Amazon have spent billions of dollars building large-scale computing systems containing a collection of hundreds of thousand computers. The commercialisation of these systems means that computing can be delivered on-demand. The scale of operation of cloud computing is comparatively bigger than that of grid computing. Furthermore, this allows computing to be provided cheaply (economies of scale) than previously thought with grid computing [7].

Cloud computing has evolved through a series of phases there was the initial grid (or utility) computing phase, then there was the "application service provision" which was then followed what is now known as SaaS [18]. According to Mohammed, the most recent evolution of cloud computing is its development with Web 2.0. This was made possible as bandwidth increased in the late nineties. In 1999, salesforce.com pioneered the concept of delivering enterprise applications via a simple website. As a result, companies; both mainstreams and specialists started the delivery of Internet-based services and applications [18]. Following on from that, Amazon Web Services was developed in $2002-$ which allowed for many cloud-based services such as computing and storage to be delivered online. In 2006, Elastic Computer Cloud (EC2) was launched by Amazon. EC2 was a commercial web service which enables individuals and small companies to rent computers online to run their own applications [18]. Since 2007, cloud computing has become a "hot topic" due to its flexibility to offer dynamic IT infrastructure and configurable software services over the Internet [25]. The emergence of cloud computing coincides with the development of Virtualisation technologies. Since 2007, Virtualisation technologies have increased and as a result, cloud computing has been observed to have out-paced that of grid computing [26]. This trend still continues to grow as companies and research community propose and develop this computing paradigm - cloud computing. According to Mohammed, a great milestone of cloud computing came about in 2009 with the introduction of Web 2.0. The Web 2.0 is designed to allow the web work as a platform i.e. clients' services do not depend on the operating system (OS) being used by the user [23] The main properties of Web 2.0 are information sharing, user-centred design and interoperability - all of these are factors that have contributed to the continual development of cloud computing over the last few years. 


\section{TECHNICAL ISSUES OF CLOUD COMPUTING}

The idea behind cloud computing is that software and hardware services are stored in "clouds", web servers rather than a connection of standalone computers over the Internet. Here, a user can access the right services and data they require [2]. Another benefit of cloud computing is that of "moving" data to the cloud to allow for access to a user's data anywhere [2]. An important feature that comes with cloud storage of data is essentially the automation of different management tasks.

It can be noted that a fusion or combination of technologies such as grid computing, autonomic computing (AC) and utility computing has contributed to the evolution of cloud computing. AC is built on the following concepts; selfprotection, self-management, healing and configuration. It uses a closed control loop system which allows it monitor and control itself with external input. As the current situation and needs of a system changes, an AC system adapts itself to those dynamical changes - making it self-adaptive as well. This combined with grid computing which was known to be "heterogeneous and geographically detached" [2], has produced a new computer architecture for cloud computing.

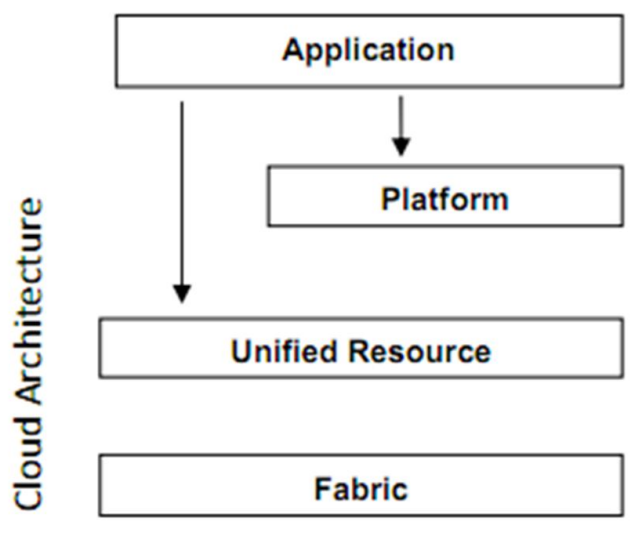

Figure 2: The Cloud Computing Architecture

[7]define a four-layer architect of cloud computing (Figure 2). These layers are Application, unified resource, platform and fabric. The physical hardware resources (such as storage resources, computing resources and network resources are contained in the Fabric layer. Abstracted/encapsulated resources (usually as a result of virtualisation) are contained in the Unified Resource layer. These abstracted resources are exposed to the upper layer and also by the end user as integrated resources such as a database system, logical file system, a virtual cluster/computer [7]. Specialised tools and technologies such as middleware and other services are provided by the Platform layer in addition to the resources already contained in the unified resource layer to provide a platform for the development and deployment of applications. Lastly, applications that will run in the cloud are contained in the Application layer. There are three different levels of services that are provided by cloud computing (IaaS, PaaS and SaaS). The type of service provision depends on the layer which the service provider wants to make available. However, it is also possible for a service provider to expose services on more than one layer.
IaaS allows for the provision of software, hardware and equipment usually at the unified resource layer. PaaS provides users with a high-level integrated environment to build, test, and deploy their own built applications. Here, developers are faced with certain technical limitations and restrictions on the type of software tool or application that they can develop in exchange for built-in application scalability [7]. The Google App Engine now enables users to build web application by using the same systems that Google uses to run its application. SaaS, on the other hand, enable service providers to provide specifically built applications and software that can be accessed remotely by end users via the Internet using a utility or usage-based model of pricing [7].

Here, the main security issue is that of the openness of the hosting or services providers. Building a test environment in the cloud requires hosted compilers which can provide a gateway for "hacker" and experience programmer alike to develop and deploy malicious programs in the cloud. A possible way to deal with this security threat for cloud provides to accept pre-compiled programs that are thoroughly scanned for viruses before being deployed. This can be achieved by restricting users to deploying programs only in the application layer - thereby restricting the risk of contamination across layers within the cloud.

Furthermore, the provision of services at different levels brings about the need for standards to be defined to allow for the exchange of information and services between clouds. To date, such standards do not exist that cause interoperability issues. There is always a security concern when standards and protocols are not properly defined in any computing environment. As cloud continues to grow and mature, there will be the need to adopt industry-wide standards that will ease interoperability issues and increase the levels of services that cloud provider can deliver to end users.

There are numerous security concerns when it comes to software development over cloud computing. This makes it difficult for certain computing techniques to be incorporated in software development. Additionally, some techniques make programs or software vulnerable in distributed systems and in this scenario, cloud computing.

\section{CROSS-CUTTING CONCERN}

Cross-cutting concerns in software development relates to aspects of a program that affect or crosscut other modules or concerns [1]. Usually, these concerns arise due to difficulty in decomposing them from a program in the developmental stage, which includes the design, coding and implementation phases, as a result can occur in the duplication of code (known as scattering) or tangling (these come about when systems have significant dependence on each other) or both . Some examples of cross-cutting concerns include:

- $\quad$ Exception handling

- Validation

- $\quad$ Logging

- Authentication and authorisation

A suggested way to deal with cross-cutting concerns in program development is to use Aspect-Oriented Programming (AOP). Aspects relates to a feature or part of a program that is not linked to the core functionality of the program but linked to many other parts of the program. Using separation of concerns (SoC), cross-cutting can be 
reduced or eliminated from program development - this is the basis of AOP. SoC is a process of distinctly separating functions of a program to avoid or limit functionality overlapping. Traditionally, SoC was achieved mainly by encapsulation and modularisation. AOP aims to increase modularity by enabling SoC. It also requires programs to be broken in distinct logical parts $-\mathrm{SoC}$ called concerns.

Developing programs on cloud computing can be done using AspectJ - a java extension which is known as the de facto standard development tool for AOP- to ease cross-cutting worries [16]. It can be challenging to develop a program on cloud as it might difficult to ascertain how to break down a program into logical parts on different servers.

\section{PROGRAM SLICING}

Another technique of software development is that of program slicing. Program slicing relates to the simplification of programs by focusing on selected aspects of semantics. The process of program slicing involves the deletion of certain parts of a program that are known to have no impact or effect on a particular semantics. Program slicing also allows developers to focus more attention of the parts of the program that can cause a fault. As a result, there are application of program slicing in testing and debugging, program comprehension, software re-engineering and measurement [10].

There are mainly two dimensions to program slicing; the semantic dimension and the syntactic dimension. The preservation of parts of the program relates to the semantic dimension. Here, the static behaviour of the program is unaffected after slicing and likewise, dynamic criteria enable the dynamic behaviour of the system to be preserved. Under the semantic dimension, slicing can be dynamic, static or conditioned [10]. However, there is less choice under the syntactic dimension. Here, there are two main possibilities; firstly the syntax of an original program is preserved, where possible, by moving parts of the programs which does not affect the interested semantic, secondly program slicing is freely allowed to perform any syntactic transformation that preserves semantic conditions - this is known as amorphous slicing [10].

Program slicing could have issues with regards to cloud computing. Deleting of certain parts of the program on clouds can affect other applications. Additionally, parts of a program that are thought of having no impact on core semantic of a program on one server could have a bigger impact on a program on another server.

\section{PROGRAM OR APPLICATION CLUSTERING}

Clustering, in computing, relates to a group of computers or servers dedicated to performing a single task. Software systems are used to configure servers to cluster in application clustering. Servers are connected together by a software program which enables the servers to perform individual tasks like failure detection and load balancing [4]. Here, applications are installed individually on the servers and are pooled in together to handle various tasks when required. It becomes important for the cluster to effectively handle routing of data to and from the cluster [4].

In cloud computing, program clustering helps achieve scalability - the ability of the cloud to appropriate resources to specific tasks i.e. when a task needs more computing resources, it has the ability to recruit more servers or computing power to perform that specific task. The benefit of cloud computing is that it contains hundreds of thousands of connecting computers which makes it easy to distribute work load. There are symmetric clusters where workload is distributed evenly amongst the clustering servers and asymmetric clusters have the ability to reserve particular servers only for use when the main servers fail. Cloud computing provides a single point of access to its end users to gain access to application services stored on the servers in the "cloud". Servers can fail; as a result clouds must tackle the issue of passing tasks around when servers failed.

\section{NON-TECHNICAL ISSUES OF CLOUD COMPUTING}

Cloud computing comes with other non-technical issues or concerns which if not tackled could restrict the growth and evolution of cloud computing.

\section{INADEQUATE SECURITY}

Most cloud vendors support what is known as multi-tenancy compute environment by design. What is most important is that, vendors must decide on the right balance between providing essential infrastructure and internal security and the quest for improved cloud computing services. According to [27], trustworthiness is important when it comes to SaaS services. With SaaS, data privacy and security are the most important factors for end users (also known as tenants).

\section{LACK OF COMPUTABILITY WITH EXISTING APPLICATIONS}

Another major issue currently facing cloud computing is the lack of inherent computability with existing applications. There are, however, efforts to change this. What is observed in order to improve scalability and improve the level of services provided to users, vendors are now providing snippets of existing codes in the case of PaaS. What this means is that new applications are becoming cloud-specific.

\section{LACK OF INTEROPERABILITY BETWEEN CLOUDS}

The lack of a standardisation across platform increases cost of switching clouds and also increases the complexity of code in the event of program migration. Since cloud vendors have different application models, there are vertical integration problems which make it virtually impossible at time to move from one cloud to another. As this is major issue, a user has to be careful when choosing the right vendor to obtain services from.

\section{OTHER ISSUES}

There is also the issue of service legal arrangement which prohibits a user from moving from one cloud to another unless certain conditions are met. This increases switching costs for the end user and subsequently, gives more power to the cloud vendor.

\section{LEGAL ISSUES}

According to [17], the biggest issue concerning cloud computing comes from governments. This is a result of the borderless global network operations of cloud computing. Unlike grid computing, cloud computing is not geographicspecific. Having no borders makes it difficult for 
governments to protect or control how data of its people is stored or used elsewhere and also how to tax companies operating services over a cloud. Under taxation, if a company is taxed based on geographical location of its computing operation, it can simply move this to a virtual office in a country with a lower tax rate [17].

There are measures being taken to tackle the issue of taxation under cloud computing on a global approach in order to stop companies from exploiting tax advantages. Additionally, there is a recognised need for harmonised laws in the global front to police how data is stored and used over the cloud.

\section{SECURITY ISSUES}

According to [11], one of the main security concerns of cloud computing is that of its immaturity of the technology. The cloud provider and client have both security responsibilities depending on the type of service. In the case of an IaaS service model, the virtualization software security, environment security and physical security rest with the cloud provider. The client or user is responsible for operating system, data and applications. However, in a SaaS model, software services, physical and environment security are the responsibility of the cloud provider.

The main security concern with cloud computing is that of data security. Confidential documents stored on the cloud can become vulnerable to attacks or unauthorised access or modification. There is also the issue of where the data is physically stored i.e. where the data stores are located. Some companies prohibit the storage of their data in certain jurisdictions or countries [13]. Here, trust in cloud computing is very vital in ensuring that data is properly managed as depending on the type if model adopted, IaaS, SaaS or PaaS, the governance of applications and data lies outside the control of the owner [6]. A possible address to this security issue is to use an Active Directory ( $\mathrm{AD}$ or LDAP) in authenticating users who can have access to data and applications in the cloud. Using Access Control Lists (ACLs), permissions can be assigned per document stored and restrict users from unauthorized access and modification. Additionally, there are now various security software tools which can be deployed in the application layer to provide and enhance authentication, operational defence, confidentiality and message integrity [12].

There are numerous encryption techniques that have been developed to further to ensure that data is securely stored in the cloud. In [14], the authors used an encryption technique known as "Elliptic curve cryptography encryption" in an attempt to protect and make secure, data stored in the cloud. According to [20], clouds are constantly being attacked, on a daily basis, and as such extra security protocols are needed to ensure security integrity. The authors proposed the use of "Transparent Cloud Protection System (TCPS)" to increase cloud security. Although, their system provided increased virtualization and transparency, it was never tested in a professional cloud environment and as such makes it difficult to establish how useful such a system really is.

Another possible way to address security issues in the cloud is to use Trusted Third Part (TTP) services within a particular cloud. Here, TTP established trusted and secure interaction between trusted parties in the cloud. Any untrusted party, can simply be ignored or blocked from access data and application within that cloud. TTP can be useful in ensuring confidentiality; authenticity and integrity are maintained in the cloud [6].

The major security worry is that most concerns and issues discussed in this review are looked at the problems in isolation. However, according to [22] the technical and security issues of cloud computing need to analysed together to gain a proper understanding of the widespread threat of the "cloud".

\section{APPLICATIONS OF CLOUD COMPUTING}

One of the reasons for the upward trend of resources committed to cloud computing is that, cloud computing has many real benefits and applications to companies, individuals, research bodies and even government. As the size of data increases, the need for computing power capable of analysing these data increases relatively.

One application of cloud computing is that clients can access their data and applications from anywhere at any particular time. Additionally, clients only need a computing device and an Internet connection to access their data and applications. For example, Dropbox [21] allows users to store their data on an online cloud and access it using any computing device. Users can also share folders with other users in the same manner. Another example is Google Docs [21] which allows users to edit, modify documents online without having to move the documents around. All modifications are saved on the master document on the cloud.

Cloud computing has the possibility of reducing hardware costs for many companies and individuals. Clients can gain access to faster computing power and bigger storage without paying for the physical hardware.

Cloud computing gives companies the ability to gain company-wide access to its host of software or applications. Here, a company does not have to buy a licence for every employee to use particular software; rather it can pay a cloud computing company on a usage fee basis (utility computing model). With the introduction of Web 2.0, access to cloud computing has become less OS-dependent. [21].

\section{CONCLUSION}

Cloud Computing describes the provision and delivery of services that are hosted over the internet according to [24]. The infrastructure of cloud computing allows services and applications to be provided and accessed from anywhere in the world as services are offered through data centres [19]. Here, the cloud becomes the single access point for customers/users to access services.

The number of reasons have contributed to the success of cloud computing over the last few years. One reason being the significant improvement of innovations in distributed and virtualisation computing. Furthermore, cheaper access to high-speed internet has also contributed to this accelerated interest [18].

There are three main categories of service provision in cloud computing are Software-as-a-Service (SaaS), Platform-as-aService (PaaS) and Infrastructure-as-a-Service (IaaS). In SaaS cloud model, the hardware infrastructure and the provision of the software product is supplied by the service provider supplies. PaaS is described as "a set of software and 
product development tools hosted on the provider's infrastructure." Developers are able to develop and create software tools over the internet and also on the provider's platform using APIs providers by the cloud provider. IaaS providers "provides virtual server instance API to start, stop, access and configure their virtual servers and storage" [24].

However, there are technical issues that need to be considered carefully when cloud computing is concerned one of them being the use of program clustering. Program clustering helps achieve scalability - the ability of the cloud to appropriate resources to specific tasks i.e. when a task needs more computing resources, it has the ability to recruit more servers or computing power to perform that specific task. This requires the need for a management system in recruiting servers and one that is able to prevent bottlenecks when servers fail. Program slicing allows for the simplification of programs by focusing on selected aspects of semantics. Deleting parts of a program that are thought of having no impact on core semantic of a program on one server could have a bigger impact on a program on another server. It can be challenging to develop a program on cloud as it might difficult to ascertain how to break down a program into logical parts on different servers.

In addition to technical issues, there are real security issues with regards to cloud computing. The most concerning security issue is that of data privacy and integrity in the cloud. However, there are many works and techniques being developed to combat the threat of data and application misuse and access in the cloud. The issues, both technical and security related, have all been observed in isolation. What must be noted is that, when these issues are combined, it could be a huge threat to cloud computing and such it is imperative that the issues be addressed not in isolation.

One of the reasons for the upward trend of resources committed to cloud computing is that, cloud computing has many real benefits and applications to companies, individuals, research bodies and even government. As the size of data increases, the need for computing power capable of analysing these data increases relatively. Another application of cloud computing is that user can access their data and applications from anywhere at any particular time.

As cloud computing continues to grow, so we hope the technical issues and as the need for standardisation to allow clouds to exchange information effectively and concisely. Also the current well established cloud vendors to be prepared to get rid of their standards.

\section{ACKNOWLEDGEMENTS}

Our thanks to the colleague Lecturers of Computer Science department Federal College of Education (Technical) Potiskum for their contributions towards development of the paper.

\section{REFERENCES}

[1] Abdullin, R. (2010) "Cross-cutting concern". From: http://abdullin.com/wiki/cross-cutting-concern.html, Accessed 11 ${ }^{\text {th }}$ Feb 2013.

[2] Aymerich, F., Fenu, G. and Surcis, S. (2008). “An Approach to a Cloud Computing Network". First International Conference on the Applications of Digital Information and Web Technologies, ICADIWT.
[3] Biddick, M. (2010). "Why You Need a Saas Strategy". Retrieved February 12, 2013, from:

http://www.informationweek.com/news/services/saas/showA rticle.jhtml?articleID=222301002

[4] Bliss, H. (2010). "What is Application Clustering?" Available at: http://www.wisegeek.com/what-is-applicationclustering.htm, Accessed $14^{\text {th }}$ Feb 2013

[5] Brooks, F. (1997). "The Mythical Man-

Month". Addison-Wesley.

[6] Dimitirios, Z. and Dimitrios, L. (2012) Addressing cloud computing security issues. Elsevier, 28 (3), p.583-59, Available at:

http://www.sciencedirect.com.ergo.glam.ac.uk/science/articl e/pii/S0167739X10002554. Accessed: 10thFeb, 2013.

[7] Foster, I., Zhao, Y., Raicu, I. and Lu, S. (2008) "Cloud

Computing and Grid Computing 360-Degree Compared"

[Online Article] from:

http://arxiv.org/ftp/arxiv/papers/0901/0901.0131.pdf,

Retrieved 14 Feb 2013.

[8] Gaw, P. (2008). "What's the Difference between Cloud

Computing and SaaS". Available at:

http://cloudcomputing.sys-con.com/node/612033,

Accessed on 11 Feb 2013.

[9] Gu, L. and Cheung, S-C. (2009). "Constructing and

Testing Privacy-Aware Services in a Cloud Computing

Environment - Challenges and Opportunities". Internetware.

ACM 978-1-60558-872-8/10.

[10] Harman, M. and Hierons, R.M. (2006). "An Overview

of Program Slicing”. Available at:

http://www.cs.ucl.ac.uk/staff/mharman/sf.html, Retrieved on: 10 $0^{\text {th }}$ Feb. 2013.

[11] Hocenski, Ž. and Kresimir, P. (2010) "Cloud computing security issues and challenges ", paper presented at MIPRO, 2010 Proceedings of the 33rd International Convention, 2428 May. IEEE Conference Publications, p. 344 - 349.

Available at:

http://ieeexplore.ieee.org.ergo.glam.ac.uk/stamp/stamp.jsp?tp $=\&$ arnumber $=5533317$, Accessed $15^{\text {th }}$ Feb. 2013.

[12] Karadesh, L. (2012) Applying Security Policies and service level Agreement to Iaas service Model to Enhance Security and Transition. Elsevier, 31 (3), p.315-326.

Available at:

http://www.sciencedirect.com.ergo.glam.ac.uk/science/articl e/pii/S0167404812000077, Accessed: $12^{\text {th }}$ Feb, 2013.

[13] King, N. and Raja, V. (2012) Protecting the privacy and security of sensitive customer data in the cloud. Elsevier, 28 (3), p.308-319. Available at:

http://www.sciencedirect.com.ergo.glam.ac.uk/science/articl e/pii/S0267364912000556, Accessed: 10th Feb, 2013.

[14] Kumar, A. et al. (2012) "Secure Storage and Access of Data in Cloud Computing", paper presented at ICT

Convergence (ICTC), 2012 International Conference on, 1517th Oct.. IEEE Conference Publications, p.336 - 339. 
International Journal of Computer Applications Technology and Research

Volume 3- Issue 3, 169 - 175, 2014, ISSN: 2319-8656

Available at:

http://ieeexplore.ieee.org.ergo.glam.ac.uk/stamp/stamp.jsp?tp $=\&$ arnumber $=6386854$, Accessed: $12^{\text {th }}$ Feb, 2013.

[15] Lewis, C. (2009). "Infrastructure as a Service".

Available at: http://clouddb.info/2009/02/23/defining-cloudcomputing-part-6-iaas/, Accessed: $6^{\text {th }}$ Feb 2013.

[16] Li, S. (2005). “An Introduction to AOP”. Available at: http://www.ibm.com/developerworks/java/tutorials/jaopintro/section4.html [Accessed $12^{\text {th }}$ Feb 2013].

[17] Lonbottom, C. (2008) "Obstacles to Cloud Computing".

[Online] Available at: http://www.information-

management.com/news/10002177-1.html?pg=1, Accessed

$11^{\text {th }}$ Feb 2013.

[18] Mohammed, A. (2009). "A history of cloud computing",

Available at:

http://www.computerweekly.com/Articles/2009/06/10/23542

9/A-history-of-cloud-computing.htm, Retrieved: 7th Feb

2013.

[19] Schneider, L. (2011). "What is cloud computing?"

Available at:

http://jobsearchtech.about.com/od/historyoftechindustry/a/cl oud_computing.htm Accessed on 10 ${ }^{\text {th }}$ Feb 2013.

[20] Shaikh, F. and Haider, S. (2011) "Security threats in cloud computing ", paper presented at 6th International

Conference On Internet Technology And Secured

Transactions, Abu Dhabi, 11-14th Dec. IEEE Conference

Publications.
[21] Strickland, J. (2011) "How Cloud Computing Works"

[Online] Available

http://computer.howstuffworks.com/cloud-computing2.htm Accessed 14 ${ }^{\text {th }}$ Feb 2013.

[22] Sun, D. et al. (2011) Addressing cloud computing security issues. Elsevier, 15 p. 2852-2856. Available at: http://www.sciencedirect.com.ergo.glam.ac.uk/science/articl e/pii/S1877705811020388 Accessed: $12^{\text {th }}$ Feb, 2013.

[23] TechPluto (2009). "Core Characteristics of Web 2.0

Services". Available http://www.techpluto.com/web-20-

services/ [Accessed 10 ${ }^{\text {th }}$ Feb 2013].

[24] TechTarget (2007). "Cloud Computing" Retrieved from: http://searchcloudcomputing.techtarget.com/definition/cloudcomputing, Accessed on: 12 $2^{\text {th }}$ Feb 2013.

[25] Wang, C. et al. (2008) "Privacy-Preserving Public Auditing for Data Storage Security in Cloud Computing", paper presented at INFOCOM, 2010 Proceedings IEEE, IEEE Conference Publications, p.1-9.[online] Available at: http://ieeexplore.ieee.org.ergo.glam.ac.uk/stamp/stamp.jsp?tp $=\&$ arnumber $=5462173$, Accessed on: $12^{\text {th }}$ Feb 2013.

[26] Wang, L. et al. (2018) Cloud Computing: A Perspective Study. New Generation Computing, Springer Link, 28 (2),

P.137-146

[27] Zhang Q., Cheng L., and Boutaba R., (2009) Cloud

Computing: State of the art and research challenges $\mathbf{J}$ internet Serv Appl 1 Brazilian Computer Society pg. 7 - 18. 